\title{
Simple and Fast Approach for Synthesis of Reduced Graphene Oxide-MoS 2 Hybrids for Room Temperature Gas Detection
}

\author{
Rajesh Kumar ${ }^{\circledR}$, Wagner Dias, Jr., Rafael J. G. Rubira, Andrei V. Alaferdov, Alfredo R. Vaz, \\ Rajesh K. Singh, Silvio R. Teixeira, Carlos J. L. Constantino, \\ and Stanislav A. Moshkalev, Member, IEEE
}

\begin{abstract}
The combination of two highly versatile materials as reduced graphene oxide (rGO) and molybdenum disulfide $\left(\mathrm{MoS}_{2}\right)$ forms the layered $\mathrm{rGO}^{-\mathrm{MoS}_{2}}$ hybrids that have great potential for sensing applications. In this paper, we developed a cost-effective, time-saving, and efficient microwave-assisted method to exfoliate rGO and $\mathrm{MoS}_{2}$ nanosheets in a powder mixture for the formation of rGO-MoS 2 hybrids. The formation of hybrids with a combination of organic and inorganic 2-D layered materials offers new possibilities for the development of gas sensitive materials. The applied microwave treatment is a simple and fast process for the large-scale synthesis of rGO-MoS ${ }_{2}$ hybrids. The synthesized rGO-MoS ${ }_{2}$ hybrids were characterized by $X$-ray diffraction, scanning electron microscope, energy dispersive X-ray spectroscopy, Raman, X-ray photoelectron spectroscopy, and thermogravimetric analyses to determine the phase structure, surface morphology, defect formation, binding energy, thermal stability and so on. The synthesized rGO-MoS ${ }_{2}$ hybrids were tested for sensing application and showed the good performance to detect gases such as $\mathrm{O}_{2}$, $\mathrm{N}_{2}$, and $\mathrm{NH}_{3}$ at room temperature.
\end{abstract}

Index Terms-2-D layered materials, characterization, gas sensor, hybrids, microwave exfoliation, molybdenum disulfide $\left(\mathrm{MoS}_{2}\right)$, reduced graphene oxide (rGO).

Manuscript received May 11, 2018; revised June 22, 2018; accepted June 27, 2018. Date of publication July 19, 2018; date of current version August 21, 2018. This work was supported in part by FAPESP (2013/14262-7 and 2017/22186-0) and in part by CNPq, Brazil. The review of this paper was arranged by Editor F. Schwierz. (Corresponding authors: Rajesh Kumar; Stanislav A. Moshkalev.)

R. Kumar was with the Centre for Semiconductor Components and Nanotechnology, University of Campinas, Campinas 13083-870, Brazil. $\mathrm{He}$ is now with the Faculty of Science and Technology, Department of Physics, São Paulo State University, Presidente Prudente 19060-900, Brazil (e-mail: rajeshbhu1@gmail.com).

W. Dias, Jr., R. J. G. Rubira, S. R. Teixeira, and C. J. L. Constantino are with the Faculty of Science and Technology, Department of Physics, São Paulo State University, Presidente Prudente 19060-900, Brazil (e-mail: snow_dias@ hotmail.com; rafael.gon.fis@gmail.com; rainho@fct.unesp.br; carlos.constantino@unesp.br).

A. V. Alaferdov, A. R. Vaz, and S. A. Moshkalev are with the Centre for Semiconductor Components and Nanotechnology, University of Campinas, Campinas 13083-870, Brazil (e-mail: analaferdov@gmail.com; alfredo@ccs.unicamp.br; stanisla@unicamp.br).

R. K. Singh is with the School of Physical and Material Sciences, Central University of Himachal Pradesh, Dharamshala HP-176215, India (e-mail: rksbhu@gmail.com).

Color versions of one or more of the figures in this paper are available online at http://ieeexplore.ieee.org.

Digital Object Identifier 10.1109/TED.2018.2851955

\section{INTRODUCTION}

$\mathbf{T}$ HE discovery of 2-D graphene and its excellent properties has encouraged numerous studies for various kinds of applications. Also, the derivatives of graphene, such as graphene oxide $(\mathrm{GO})$ and reduced $\mathrm{GO}(\mathrm{rGO})$, have recently concerned remarkable research curiosity owing to their unique structural and outstanding electronic, mechanical, and thermal properties. The GO/rGO layers contain different functional groups such as hydroxyl and epoxy groups on $\mathrm{sp}^{3}$ hybridized carbon on the basal plane, in addition to carbonyl and carboxyl groups located at the sheet edges on $\mathrm{sp}^{2}$ region [1]. These oxygen-containing functional groups attached to $\mathrm{GO} / \mathrm{rGO}$ makes it more sensitive, and its degree of sensitivity depends on these attached functional groups [2]. Due to nearly absence of bandgap in graphene and its derivatives, there are numerous efforts to find out other types of 2-D layered inorganic materials such as transition metal dichalcogenides (TMDs). In the category of TMDs, molybdenum disulfide $\left(\mathrm{MoS}_{2}\right)$ contains graphene-liked 2-D layered n-type semiconductor, which composed of three atom layers (S-Mo-S) and also stacked through a weak Van der Waals force [3]. These $\mathrm{MoS}_{2}$ layered materials have stimulated intensive research due to their outstanding semiconducting nature with unique thickness-dependent physical properties which are used for versatile electronic, optical, and sensing applications.

Several research studies on rGO-based gas sensors are discussed and show the potential of rGO in sensing application [4]-[6]. The rGO-based sensors are capable of detection of chemically aggressive gases, such as $\mathrm{NO}_{2}, \mathrm{NH}_{3}$, $\mathrm{Cl}_{2}$, and $\mathrm{NO}$ with the typical response and recovery times of about several tens of minutes [4], [7]-[10]. Also, the assembled structure graphene into a 3-D graphene aerogel may open up new prospects for improved sensing properties by maintaining a high surface area in a porous network [11]-[13]. However, graphene and its derivatives-based sensors show a poor selectivity without proper surface modification [10], [14]-[17]. Therefore, it is remarkably important to develop high performance containing gas sensors that are capable of sensing low concentrations of gases in atmosphere precisely, constantly, and rapidly for human health safety 
and air-quality monitoring [18]. The single-/few-layered $\mathrm{MoS}_{2}$ shows improved selectivity compared to graphene, makes an exciting candidate for gas sensing application including at a room temperature. Single/few layers synthesized $\mathrm{MoS}_{2}$ has been used as a prospective sensing material for several kinds of gases [3], [18]-[25]. The $\mathrm{MoS}_{2}$-based field-effect transistor sensors have slow response and recovery times or even no recovery when used at normal room temperature [26]-[31].

Graphene with single-/few-layer $\mathrm{MoS}_{2}$ nanosheets $\left(\mathrm{MoS}_{2}\right.$ $\mathrm{NSs}$ ) reveals enhanced excellent properties and offers the possibilities to create new kinds of devices for daily human life such as supercapacitors, lithium storage, and DNA sensing [32]-[37]. The combined structure of graphene/rGO with $\mathrm{MoS}_{2}$ shows a good application for gas sensing. For conductometric sensing purposes, the graphene derivatives allow for lower noise measurements than $\mathrm{MoS}_{2}$ alone, since $\mathrm{MoS}_{2}$ is much less conductive than graphene due to its semiconducting nature [37]. The 2-D structure of the $\mathrm{MoS}_{2}$ on graphene/rGO increases the contact area for efficient charge transfer across the interface and shortens the charge transport time and distance, which improve the device performance [35], [36], [38]. Also, the formation of hybrids with the combination of graphene/rGO- $\mathrm{MoS}_{2}$ architectures can improve their electrochemical, catalytic, and sensing behaviors [33], [39]. Niu et al. [3] have reported the synthesis of $\mathrm{rGO} / \mathrm{MoS}_{2}$ composite using two-step process (wet spinning and hydrothermal) for $\mathrm{NO}_{2}$ and $\mathrm{NH}_{3}$ gas sensing. Cho et al. [40] have developed atomically thin heterostructure-based gas sensor via the combination of $\mathrm{MoS}_{2}$ (mechanically exfoliated) and graphene (synthesized via chemical vapor deposition) for the detection of $\mathrm{NO}_{2}$ gas.

In this paper, we have developed one-step, simple, rapid, and large-scale synthesis using microwave-assisted $\mathrm{rGO}-\mathrm{MoS}_{2}$ hybrids for application at room temperature gas $\left(\mathrm{O}_{2}, \mathrm{~N}_{2}\right.$, and $\mathrm{NH}_{3}$ ) sensors. The fast chemical reactions on $\mathrm{rGO}-\mathrm{MoS}_{2}$ hybrids surfaces using this method enable reducing production costs and enhancing sensing capabilities. These studies revealed the potential application of $\mathrm{rGO}-\mathrm{MoS}_{2}$ hybrids for a different gas sensing.

\section{EXPERIMENTS}

\section{A. Synthesis of rGO Nanosheets}

Graphite oxide was synthesized by the chemical oxidation of graphite powder, using the modified Staudenmaier's method [41], [42]. Graphite powder (5 g) was continuously stirred with a combination of sulfuric acid $(90 \mathrm{~mL})$ and nitric acid (45 mL) solution at room temperature. The solution container was placed into an ice water bath to make sure constant temperature and subsequently, potassium chlorate $\left(\mathrm{KClO}_{3}\right)$ $(40 \mathrm{~g})$ was gradually poured into the solution to reduce the risk of explosion due to the exothermic reaction. This solution was kept for 6 days under continuous magnetic stirring at room temperature for superior oxidation of the graphite powder. The as-obtained graphite oxide product was washed with deionized water (DI) water and further $10 \%$ hydrochloric solution was added to eliminate sulfate and other ion impurities. It was then again washed several times with DI water until a pH of 7 was reached. Afterward, the graphite oxide powder was dried at 60
${ }^{\circ} \mathrm{C}$ under vacuum for complete dry. The dried graphite oxide powder was directly irradiated $(700 \mathrm{~W}$ and $70 \mathrm{~s})$ in a domestic microwave oven (Consul-CMW30AB, 1-kW total power) for reduction and exfoliation into $\mathrm{rGO}$.

\section{B. Synthesis of rGO-MoS 2 Hybrids}

The graphite oxide suspension was obtained by mixing $100 \mathrm{mg}$ of graphite oxide powder in $120 \mathrm{~mL}$ of $\mathrm{C}_{2} \mathrm{H}_{5} \mathrm{OH}$ assisted with continuous magnetic stirring (20 min) and sonication. To prepare the $\mathrm{rGO}-\mathrm{MoS}_{2}$ hybrids, $\mathrm{MoS}_{2}$ powder (50 mg) was added into the as-prepared graphite oxide suspension under continuous stirring for $20 \mathrm{~min}$. Finally, the prepared solution was dried in open atmosphere for complete evaporation of $\mathrm{C}_{2} \mathrm{H}_{5} \mathrm{OH}$ and dried powder was treated with microwave (Consul-CMW30AB) irradiation (800 W and $130 \mathrm{~s}$ ) for complete exfoliation and reduction graphite oxide into $\mathrm{rGO}$ with $\mathrm{MoS}_{2}$ powder for the final formation of $\mathrm{rGO}-\mathrm{MoS}_{2}$ hybrids.

\section{Characterization}

The structural characterization of the synthesized rGO$\mathrm{MoS}_{2}$ hybrids was performed by X-ray diffraction (XRDD/MAX-2500/PC; Rigaku Corporation, Tokyo, Japan) over the $2 \theta$ range $5^{\circ}-75^{\circ}$. The surface morphology and elemental analysis were investigated using scanning electron microscope (SEM-dual beam focused ion beam/field emission gun model FEI Nova 200) equipped with energy dispersive Xray spectroscopy (EDS). Raman measurements were carried out to determine the defect inside the material using a spectrometer with a 473-nm laser (NT-MDT NTEGRA spectra). $\mathrm{X}$-ray photoelectron spectroscopy (XPS) measurements were performed using a SPECS system XR 50 X-ray source (Al $\mathrm{K} \alpha, 1486.6 \mathrm{eV}$ ) equipped Phoibos 150 hemispherical energy analyzer with MCD-9 detector. Thermogravimetric measurements were carried out with an SDT Q600 thermogravimetric analyses (TGAs) apparatus (TA Instruments, New Castle, DE, USA), and the samples were heated at a rate of $10{ }^{\circ} \mathrm{C} / \mathrm{min}$ from room temperature to $850{ }^{\circ} \mathrm{C}$ at air flow $(100 \mathrm{~mL} / \mathrm{min})$ atmosphere.

\section{Gas Sensing Measurements}

The gas sensing tests were carried out in a vacuum chamber at room temperature. In the fabrication process, the asprepared $\mathrm{rGO}-\mathrm{MoS}_{2}$ hybrid powder was used for the preparation of two types of resistive gas sensors. First, a thick-film sensitive element was prepared in the form of a disk with a diameter of $10 \mathrm{~mm}$ and a thickness of $2 \mathrm{~mm}$, with two parallel metal electrodes fixed in the opposite parts of the disk with the spacing between the electrodes about $8 \mathrm{~mm}$. The electrodes were prepared using copper wires with diameters of $0.05 \mathrm{~mm}$ covered with gold. The powder together with electrodes was pressed to form disks with a density of $1.8 \mathrm{~g} / \mathrm{cm}^{3}$. The resistance of the material between electrodes was measured to be near $20 \Omega$ and its resistivity near $4 \Omega \cdot \mathrm{cm}$. Second, a thin-film sensor was prepared by drop casting of isopropyl alcohol solution containing the powder between two parallel thin-film graphite electrodes (width of $10 \mathrm{~mm}$, the gap between 


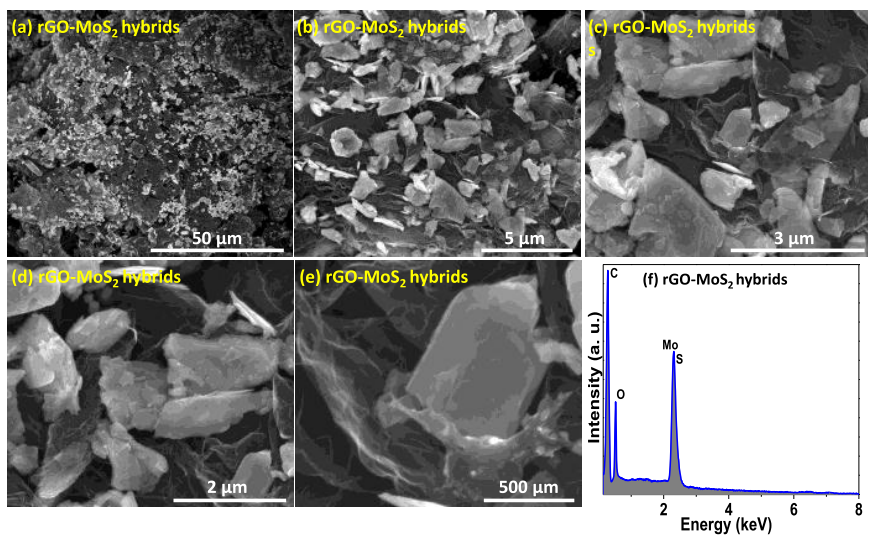

Fig. 1. (a)-(e) SEM rGO-MoS 2 hybrids at different magnifications. (f) EDS of rGO-MoS 2 hybrids.

electrodes $1 \mathrm{~mm}$ ) formed over a flexible polydimethylsiloxane support using the Langmuir-Blodgett method [43]. An estimated average thickness of the $\mathrm{rGO}-\mathrm{MoS}_{2}$ layer after drying was near $0.01 \mathrm{~mm}$, and the sensor resistance was measured to be $10 \mathrm{k} \Omega$. The prepared sensing elements were placed in a vacuum chamber with a base pressure of $10^{-5}$ mbar. The current through the sensors was maintained at $1 \mathrm{~mA}$, and the voltage drops between two electrodes were measured. The sensors were exposed to different gases as $\mathrm{N}_{2}, \mathrm{O}_{2}$, and $\mathrm{NH}_{3}$ vapor at room temperature.

\section{Results and Discussion}

The surface morphology of the $\mathrm{rGO}-\mathrm{MoS}_{2}$ hybrids was characterized using SEM. SEM micrographs of the assynthesized rGO-MoS 2 hybrids (Fig. 1) show that the synthesized materials assembled with the homogeneous distribution of rGO and $\mathrm{MoS}_{2}$ in the hybrids. The rGO, NSs, and $\mathrm{MoS}_{2}$ NSs are interconnected to each other. Specially, the porous morphology of rGO NSs gives the good supports for $\mathrm{MoS}_{2}$ NSs and it can be clearly seen in Fig. 1(e), which facilitates gas diffusion during the sensing application. The surface analysis indicates that most of the rGO NSs are covered by $\mathrm{MoS}_{2}$ NSs. The uniform distribution of $\mathrm{MoS}_{2}$ NSs on the rGO NSs is confirmed by EDS as shown in Fig. 1(f), where C, O, Mo, and $\mathrm{S}$ are seen to be uniformly distributed throughout the rGO-MoS 2 hybrids.

To determine the structural analysis, XRD patterns of the synthesized rGO NSs and rGO-MoS 2 hybrids are shown in Fig. 2. The inset of the figure shows the XRD pattern of rGO NSs, which was exfoliated and reduced by microwave irradiation. The three sharp diffraction peaks of rGO-MoS 2 hybrids center at around $2 \theta=15.1^{\circ}, 33.8^{\circ}$, and $57.5^{\circ}$ corresponding to the (002), (100), and (110) planes, respectively, for $\mathrm{MoS}_{2}$. These all of the diffraction peaks can be well indexed to the hexagonal phase of $\mathrm{MoS}_{2}$ (Joint Committee on Powder Diffraction Standards No. 37-1492) [3], [44]. The small and broad diffraction peak of rGO NSs at $2 \theta=25.2^{\circ}$ corresponding to (002) plane can be seen in the rGO-MoS 2 hybrids, suggesting the presence of rGO NSs in hybrids.

Raman spectroscopy is a powerful tool for the characterization of rGO and others 2-D layered materials [45], [46]. The

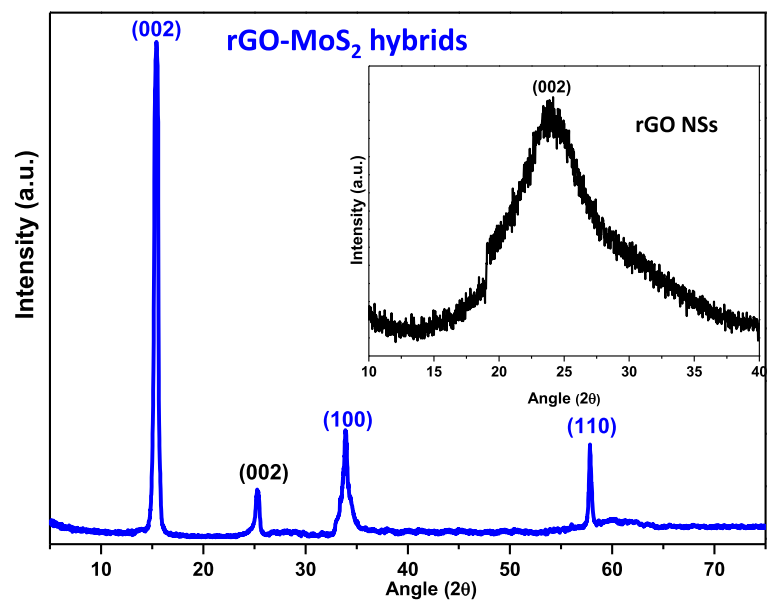

Fig. 2. XRD of rGO NSs and rGO-MoS 2 hybrids.

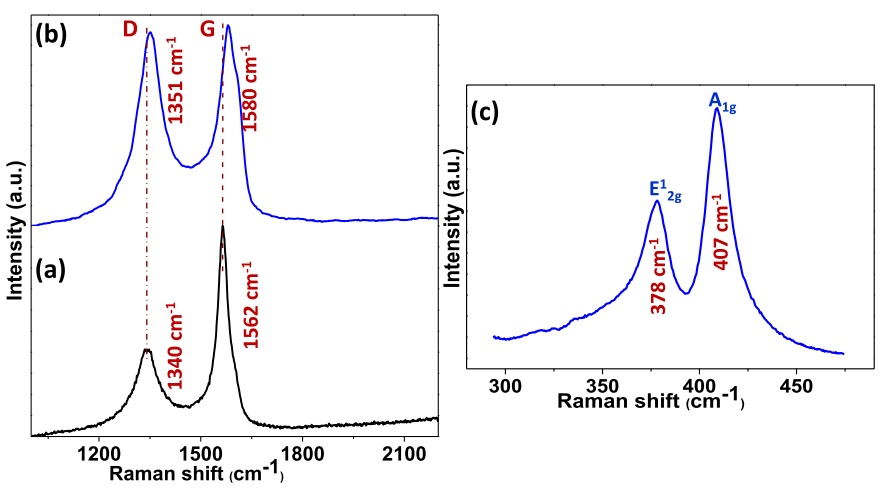

Fig. 3. Raman spectra of (a) rGO NSs and (b) and (c) rGO-MoS 2 hybrids.

Raman spectra of the as-synthesized rGO NSs and rGO--MoS 2 hybrids are shown in Fig. 3. The two dominant peaks in Raman spectrum show a typical $D$-band and $G$-band for graphene and its derivatives (rGO NSs), which present the defects or structural disorders $\left(\mathrm{sp}^{3}\right)$ and tangential vibration of carbon atoms $\left(\mathrm{sp}^{2}\right)$, respectively, [47], [48]. The $D$-band and $G$-band observed in rGO appear at 1340 and $1562 \mathrm{~cm}^{-1}$, respectively, [Fig. 3(a)]. After formation of rGO-MoS 2 hybrids, the $D$-band and $G$-band of the rGO-MoS ${ }_{2}$ hybrids observed at 1351 and $1580 \mathrm{~cm}^{-1}$, respectively, [Fig. 3(b)]. These significant shiftings were observed in the $G$-band positions in $\mathrm{rGO}-\mathrm{MoS}_{2}$ hybrids because of the interaction of $\mathrm{MoS}_{2}$. Furthermore, the broadening of Raman peaks ( $D$-band and $G$-band) for rGO- $-\mathrm{MoS}_{2}$ hybrids are the witnessed for the existence of $\mathrm{MoS}_{2}$. Also, the $I_{D} / I_{G}$ ratio can be used for the indication of the rGO-MoS ${ }_{2}$ hybrids quality. The relative intensity of $I_{D} / I_{G}$ was around 0.96 in $\mathrm{rGO}-\mathrm{MoS}_{2}$ hybrids is much higher than that in pristine rGO NSs (0.41), revealing that some defects and disordered structures such as wrinkles, holes, charged impurities, and others can be introduced on the surface of $\mathrm{rGO}$ due to the addition of $\mathrm{MoS}_{2}$. This phenomenon is supposed to be attributed to the electron transfer between rGO and $\mathrm{MoS}_{2}$. The lower wavenumber part of Raman spectra of rGO-MoS ${ }_{2}$ hybrids reveals the well-known characteristic peaks of $\mathrm{MoS}_{2}$ [Fig. 3(c)]. The major peaks associated with 

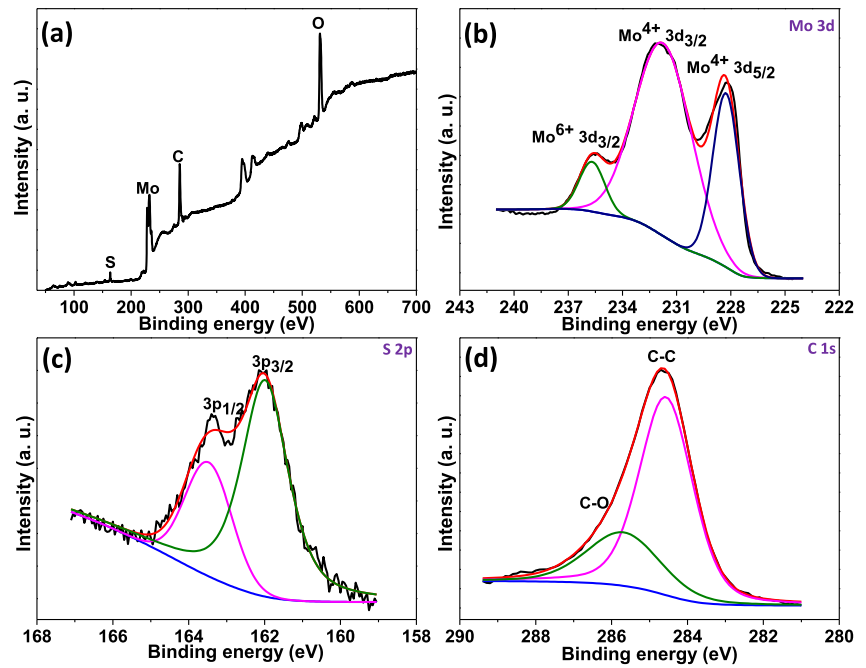

Fig. 4. XPS spectra of $\mathrm{rGO}-\mathrm{MoS}_{2}$ hybrids. (a) Complete survey, (b) Mo 3d, (c) S 2p, and (d) C1s peaks.

$\mathrm{MoS}_{2}$ are at 378 and $407 \mathrm{~cm}^{-1}$ and correspond to the in-plane $\mathrm{E}_{2 g}^{1}$ and out-of-plane $\mathrm{A}_{1 g}$ vibrational modes of hexagonal $\mathrm{MoS}_{2}$, respectively, in a good agreement with the literature values for multilayer $\mathrm{MoS}_{2}$ [3], [18], [49]. The $\mathrm{E}_{2 g}^{1}$ mode involves the in-layer relative displacement between Mo and $\mathrm{S}$ atoms, while the $\mathrm{A}_{1 g}$ mode involves the out-of-layer verticalvibration displacements of $\mathrm{S}$ atoms along the $c$-axis.

XPS is used to further study the surface electronic state of rGO-MoS 2 hybrids. The chemical composition of $\mathrm{rGO}-\mathrm{MoS}_{2}$ hybrids samples is characterized by XPS spectra. Sulfur, molybdenum, carbon, and oxygen peaks are clearly identified in the survey spectrum in Fig. 4(a). The high-resolution scan for Mo 3d, S 2p, C1s, and O1s has been shown in Fig. 4(b)-(d). The Mo peaks show two Mo oxidation states $\left(\mathrm{Mo}^{4+}\right.$ and $\left.\mathrm{Mo}^{6+}\right)$, which can be indexed to the Mo-S and the Mo-O bonding, respectively. The Mo $3 \mathrm{~d}$ spectra show two Mo oxidation states $\left(\mathrm{Mo}^{4+}\right.$ and $\left.\mathrm{Mo}^{6+}\right)$. The Mo 3d clearly shows three peaks at 228.3, 231.9, and $235.1 \mathrm{eV}$ [Fig. 4(b)]. The first two peaks (228.3 and $231.9 \mathrm{eV}$ ) are attributed to the doublet Mo $3 \mathrm{~d}_{5 / 2}$ and Mo $3 \mathrm{~d}_{3 / 2}$, respectively, correlating to $\mathrm{Mo}^{4+}$ state in $\mathrm{MoS}_{2}$ (typical of the Mo-S bond). The third peak of Mo $3 \mathrm{~d}_{5 / 2}$ at $235.1 \mathrm{eV}$ is attributed to the $\mathrm{Mo}^{6+}$ state of $\mathrm{MoO}_{3}$, typical of the Mo-O bond [50]-[52]. The formation of this kind of bond has been reported elsewhere [18], [51], [53], [54], and suggests strong chemical and electronic coupling between the $\mathrm{MoS}_{2}$ and rGO NSs in the synthesized samples. It shows the existence of Mo oxide state in the $\mathrm{MoS}_{2}$ due to the presence of oxygen in rGO NSs. In addition, the $S 2 p$ region shows two characteristic peaks [Fig. 4(c)] located at 162.0 and $163.3 \mathrm{eV}$ corresponding to $\mathrm{S} 2 \mathrm{p}_{3 / 2}$ and $\mathrm{S} 2 \mathrm{p}_{1 / 2}$, respectively, which can be indexed to Mo-S bonding in $\mathrm{MoS}_{2}$. The C1s peak [Fig. 4(d)] can be deconvoluted into two peaks, a large peak at $284.6 \mathrm{eV}$ attributed to $\mathrm{C}-\mathrm{C}$ bonding environment associated with the rGO NSs, and a smaller peak at $285.7 \mathrm{eV}$ attributed to $\mathrm{C}-\mathrm{O}$ bonding.

TGA was employed to determine the thermal stability of $\mathrm{MoS}_{2}$ in the rGO-MoS hybrids, and its weight loss curve is shown in Fig. 5. In Fig. 5, it can be observed that TGA

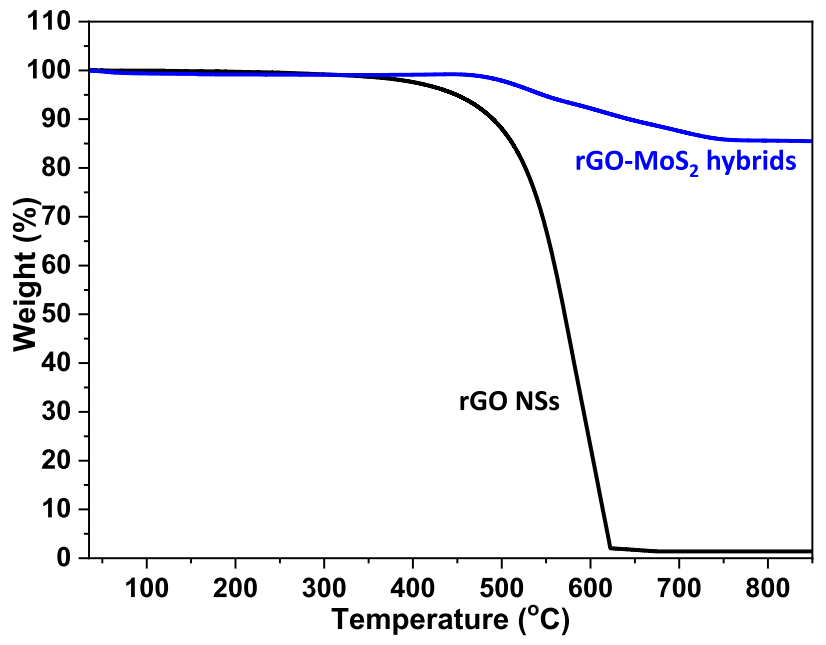

Fig. 5. TGA curve of rGO NSs and rGO-MoS 2 hybrids.

TABLE I

Sensor Response for Different Gases $\left(\mathrm{N}_{2}, \mathrm{O}_{2}\right.$, AND $\left.\mathrm{NH}_{2}\right)$

\begin{tabular}{|c|c|c|c|c|c|}
\hline $\begin{array}{c}\text { Sensor } \\
\text { type }\end{array}$ & Gas & $\begin{array}{c}\Delta U / U, \\
\%\end{array}$ & $\begin{array}{c}\mathrm{P}, \\
\mathrm{mbar}\end{array}$ & $\begin{array}{c}\tau, \\
\mathrm{sec}\end{array}$ & $\begin{array}{c}{[\Delta U / U] / \mathrm{P},} \\
\% / \mathrm{mbar}\end{array}$ \\
\hline \multirow{2}{*}{$\begin{array}{c}\text { Thick } \\
\text { film } \\
(2 \mathrm{~mm})\end{array}$} & $\mathrm{N}_{2}$ & -0.13 & 290 & 20 & $-4.4 \times 10^{-4}$ \\
\cline { 2 - 6 } & $\mathrm{O}_{2}$ & 0.34 & 5.7 & 50 & 0.059 \\
\hline \multirow{2}{*}{$\begin{array}{c}\text { Thin } \\
\text { film } \\
(0.01\end{array}$} & $\mathrm{O}_{2}$ & 0.18 & 0.48 & 15 & 0.38 \\
\cline { 2 - 6 } mm $)$ & $\mathrm{NH}_{3}$ & 0.19 & 0.008 & 8 & 24 \\
\hline
\end{tabular}

of rGO NSs and rGO-MoS 2 hybrids obviously shows two main weight losses. For rGO NSs, the weight loss starts very slowly from room temperature to around $450{ }^{\circ} \mathrm{C}(\sim 5 \mathrm{wt} \%)$, after that its decrease very rapidly and completed by $620{ }^{\circ} \mathrm{C}$ ( $\sim 98 \mathrm{wt} \%$ ). However, in the case of $\mathrm{rGO}-\mathrm{MoS}_{2}$ hybrids, the initial weight loss was very small from room temperature to $470{ }^{\circ} \mathrm{C}(\sim 1.4 \mathrm{wt} \%)$ and after that fast weight loss was observed up to $740{ }^{\circ} \mathrm{C}(\sim 15 \mathrm{wt} \%)$. These initial weight losses were likely due to the evaporation of surface absorbed water molecules and the presence of an oxygen containing functional groups. The higher weight losses were due to the decomposition and combustion of rGO NSs. For rGO-MoS 2 hybrids, the change represents its thermal decomposition and $\sim 15 \%$ weight loss in this temperature range reveals the conversion of $\mathrm{MoS}_{2}$ to $\mathrm{MoO}_{3}$ and gaseous $\mathrm{SO}_{2}$ [55]-[57].

Some preliminary results of gas sensing measurements are shown in Table I for both (thick and thin film) sensors, where $\Delta U / U$ is the sensor response (relative change of the voltage after exposure to gases with pressure $P$ ), $\tau$ is the response time, and $[\Delta U / U] / P$ is the sensitivity. In all cases, the recovery time was considerably larger than the response (rise) time, usually being in the order of hundreds of seconds compared with the rise time of a few tens of seconds. Table I contains the data for different gases $\mathrm{N}_{2}, \mathrm{O}_{2}$, and $\mathrm{NH}_{3}$ which has been used for application purposes.

As can be seen, the highest sensitivity is achieved for $\mathrm{NH}_{3}$ vapor, followed by oxygen (which is an order of 
magnitude lower). Much lower sensitivity was obtained for nonreactive gas $\mathrm{N}_{2}$. The type of response was also different for nitrogen, when the resistance of the sensing element was reduced upon exposure to the gas. In contrast, during exposure to oxygen and $\mathrm{NH}_{3}$ vapor, an increase of resistance was detected that may be attributed to the formation of negative ions like $\mathrm{O}^{-}$during gas interaction with the surface of material resulting in a decrease of measured current [58]. As no gassolid reaction is expected for nitrogen, the observed small increase of current can be probably attributed to filling the pores by gas making the whole system less resistive. Finally, the sensitivity for $\mathrm{NH}_{3}$ and $\mathrm{O}_{2}$ for a thin sensitive rGO$\mathrm{MoS}_{2}$ layer (a few parts per million) is about two orders of magnitude higher compared to the thick layer, being approximately inversely proportional to the layer thickness. This finding indicates that in the case of thick sensitive films only the top layers effectively interact with the gas, changing their resistance.

These preliminary results show great potential for the use of thin film rGO-MoS 2 hybrid material for sensing applications. Relatively high sensitivity to gases such as oxygen and $\mathrm{NH}_{3}$ vapor was achieved at room temperature, while in conventional gas sensors-based, for example, on titanium oxide, temperatures as high as $200{ }^{\circ} \mathrm{C}-300{ }^{\circ} \mathrm{C}$ are usually required to get reasonable sensitivities [58].

\section{COnClusion}

In summary, we have successfully synthesized $\mathrm{rGO}-\mathrm{MoS}_{2}$ hybrids by in situ microwave reduction and exfoliation. Our simple, fast, and low-cost synthesis approach provides a high quality of $\mathrm{rGO}-\mathrm{MoS}_{2}$ hybrids that play a good role in sensing application. Preliminary results with gas sensing $\left(\mathrm{O}_{2}, \mathrm{~N}_{2}\right.$, and $\mathrm{NH}_{3}$ ) show the great potential of a rGO-MoS 2 hybrid material for sensing applications; the highest sensitivity was achieved here for very thin sensitive layers (10 $\mu \mathrm{m}$ thick) at room temperature using a flexible polymer substrate. Further experiments are in progress aiming at the optimization of the gas sensor to achieve enhanced sensitivity and faster response and recovery times. In particular, the use of thinner layers and moderate temperatures up to $100{ }^{\circ} \mathrm{C}$ is planned to achieve smaller recovery time and more sensitive detection of gases.

\section{References}

[1] O. Akhavan, M. Kalaee, Z. S. Alavi, S. M. A. Ghiasi, and A. Esfandiar, "Increasing the antioxidant activity of green tea polyphenols in the presence of iron for the reduction of graphene oxide," Carbon, vol. 50, no. 8, pp. 3015-3025, 2012.

[2] P. Salvo et al., "Graphene-based devices for measuring pH," Sens. Actuators B, Chem., vol. 256, pp. 976-991, Mar. 2018.

[3] Y. Niu et al., "MoS 2 graphene fiber based gas sensing devices," Carbon, vol. 95, pp. 34-41, Dec. 2015.

[4] S. Basu and P. Bhattacharyya, "Recent developments on graphene and graphene oxide based solid state gas sensors," Sens. Actuators B, Chem., vol. 173, pp. 1-21, Oct. 2012.

[5] F. Yavari and N. Koratkar, "Graphene-based chemical sensors," J. Phys. Chem. Lett., vol. 3, no. 13, pp. 1746-1753, 2012.

[6] Q. He, S. Wu, Z. Yin, and H. Zhang, "Graphene-based electronic sensors," Chem. Sci., vol. 3, no. 6, pp. 1764-1772, 2012.

[7] G. Lu, L. E. Ocola, and J. Chen, "Reduced graphene oxide for roomtemperature gas sensors," Nanotechnology, vol. 20, no. 44, p. 445502, 2009.
[8] V. Dua et al., "All-organic vapor sensor using inkjet-printed reduced graphene oxide," Angew. Chem. Int. Ed., vol. 49, no. 12, pp. 2154-2157, 2010 .

[9] L. J. Cote, R. Cruz-Silva, and J. Huang, "Flash reduction and patterning of graphite oxide and its polymer composite," J. Amer. Chem. Soc., vol. 131, no. 31, pp. 11027-11032, 2009.

[10] W. Li et al., "Reduced graphene oxide electrically contacted graphene sensor for highly sensitive nitric oxide detection," ACS Nano, vol. 5 no. 9, pp. 6955-6961, 2011.

[11] M. A. Worsley, P. J. Pauzauskie, T. Y. Olson, J. Biener, J. H. Satcher, Jr. and T. F. Baumann, "Synthesis of graphene aerogel with high electrical conductivity," J. Amer. Chem. Soc., vol. 132, no. 40, pp. 14067-14069, 2010.

[12] M. A. Worsley et al., "Mechanically robust 3D graphene macroassembly with high surface area," Chem. Commun., vol. 48, no. 67, pp. 8428-8430, 2012.

[13] M. A. Worsley et al., "Synthesis and characterization of highly crystalline graphene aerogels," ACS Nano, vol. 8, no. 10, pp. 11013-11022, 2014.

[14] F. Schedin et al., "Detection of individual gas molecules adsorbed on graphene," Nature Mater, vol. 6, pp. 652-655, Jul. 2007.

[15] J. D. Fowler, M. J. Allen, V. C. Tung, Y. Yang, R. B. Kaner, and B. H. Weiller, "Practical chemical sensors from chemically derived graphene," ACS Nano, vol. 3, no. 2, pp. 301-306, 2009.

[16] T. O. Wehling, K. S. Novoselov, S. V. Morozov, E. E. Vdovin, M. I. Katsnelson, A. K. Geim, and A. I. Lichtenstein, "Molecular doping of graphene," Nano Lett., vol. 8, no. 1, pp. 173-177, Dec. 2008.

[17] A. K. Geim, "Graphene: Status and prospects," Science, vol. 324, no. 5934, pp. 1530-1534, Jun. 2009.

[18] H. Long et al., "High surface area $\mathrm{MoS}_{2}$ /graphene hybrid aerogel for ultrasensitive $\mathrm{NO}_{2}$ detection," Adv. Funct. Mater, vol. 26, no. 28, pp. 5158-5165, 2016.

[19] B. Cho et al., "Charge-transfer-based gas sensing using atomic-layer $\mathrm{MoS}_{2}$," Sci. Rep., vol. 5, Jan. 2015, Art. no. 8052.

[20] A. Shokri and N. Salami, "Gas sensor based on $\mathrm{MoS}_{2}$ monolayer," Sens. Actuators B, Chem., vol. 236, pp. 378-385, Nov. 2016.

[21] Y. Liu et al., "Hydrogen gas sensing properties of $\mathrm{MoS}_{2} / \mathrm{Si}$ heterojunction," Sens. Actuators B, Chem., vol. 211, pp. 537-543, May 2015.

[22] W. Yang, L. Gan, H. Li, and T. Zhai, "Two-dimensional layered nanomaterials for gas-sensing applications," Inorganic Chem. Frontiers, vol. 3, no. 4, pp. 433-451, 2016.

[23] D. Zhang, J. Wu, P. Li, and Y. Cao, "Room-temperature $\mathrm{SO}_{2}$ gas-sensing properties based on a metal-doped $\mathrm{MoS}_{2}$ nanoflower: An experimental and density functional theory investigation," J. Mater. Chem. A, vol. 5 , no. 39, pp. 20666-20677, 2017.

[24] F. K. Perkins, A. L. Friedman, E. Cobas, P. M. Campbell, G. G. Jernigan, and B. T. Jonker, "Chemical vapor sensing with monolayer $\mathrm{MoS}_{2}$," Nano Lett., vol. 13, no. 2, pp. 668-673, 2013.

[25] W. Di et al., "Construction of $\mathrm{MoS}_{2} / \mathrm{Si}$ nanowire array heterojunction for ultrahigh-sensitivity gas sensor," Nanotechnology, vol. 28, no. 43, p. 435503, 2017.

[26] Q. He et al., "Fabrication of flexible $\mathrm{MoS}_{2}$ thin-film transistor arrays for practical gas-sensing applications," Small, vol. 8, no. 19, pp. 2994-2999, 2012.

[27] H. Li et al., "Fabrication of single- and multilayer $\mathrm{MoS}_{2}$ film-based field-effect transistors for sensing $\mathrm{NO}$ at room temperature," Small, vol. 8, no. 1, pp. 63-67, 2012.

[28] B. Cho et al., "Bifunctional sensing characteristics of chemical vapor deposition synthesized atomic-layered $\mathrm{MoS}_{2}, "$ ACS Appl. Mater. Interfaces, vol. 7, no. 4, pp. 2952-2959, 2015.

[29] M. Donarelli et al., "Response to $\mathrm{NO}_{2}$ and other gases of resistive chemically exfoliated $\mathrm{MoS}_{2}$-based gas sensors," Sens. Actuators B, Chem., vol. 207, pp. 602-613, Feb. 2015.

[30] J.-S. Kim, H.-W. Yoo, H. O. Choi, and H.-T. Jung, "Tunable volatile organic compounds sensor by using thiolated ligand conjugation on $\mathrm{MoS}_{2}, "$ Nano Lett., vol. 14, no. 10, pp. 5941-5947, 2014.

[31] K. Lee, R. Gatensby, N. McEvoy, T. Hallam, and G. S. Duesberg, "Highperformance sensors based on molybdenum disulfide thin films," $A d v$. Mater., vol. 25, no. 46, pp. 6699-6702, 2013.

[32] K.-J. Huang, L. Wang, J.-Z. Zhang, and K. Xing, "Synthesis of molybdenum disulfide/carbon aerogel composites for supercapacitors electrode material application," J. Electroanal. Chem., vol. 752, pp. 33-40, Sep. 2015.

[33] K. Chang and W. Chen, "L-cysteine-assisted synthesis of layered $\mathrm{MoS}_{2}$ /graphene composites with excellent electrochemical performances for lithium ion batteries," ACS Nano, vol. 5, no. 6, pp. 4720-4728, 2011. 
[34] P. T. K. Loan, W. Zhang, C.-T. Lin, K.-H. Wei, L.-J. Li, and C.-H. Chen, "Graphene/ $\mathrm{MoS}_{2}$ heterostructures for ultrasensitive detection of DNA hybridisation," Adv. Mater., vol. 26, no. 28, pp. 4838-4844, 2014.

[35] K. Roy et al., "Graphene-MoS 2 hybrid structures for multifunctional photoresponsive memory devices," Nat. Nanotech., vol. 8, pp. 826-830, Oct. 2013

[36] Y. Li, H. Wang, L. Xie, Y. Liang, G. Hong, and H. Dai, "MoS 2 nanoparticles grown on graphene: An advanced catalyst for the hydrogen evolution reaction," J. Amer. Chem. Soc., vol. 133, no. 19, pp. 7296-7299, 2011.

[37] M. A. Worsley et al., "Ultralow density, monolithic $\mathrm{WS}_{2}, \mathrm{MoS}_{2}$, and $\mathrm{MoS}_{2}$ /graphene aerogels," ACS Nano, vol. 9, no. 5, pp. 4698-4705, 2015.

[38] B. Radisavljevic, A. Radenovic, J. Brivio, V. Giacometti, and A. Kis, "Single-layer $\mathrm{MoS}_{2}$ transistors," Nat. Nanotech., vol. 6, pp. 147-150, Jan. 2011.

[39] X. Zheng, J. Xu, K. Yan, H. Wang, Z. Wang, and S. Yang, "Spaceconfined growth of $\mathrm{MoS}_{2}$ nanosheets within graphite: The layered hybrid of $\mathrm{MoS}_{2}$ and graphene as an active catalyst for hydrogen evolution reaction," Chem. Mater, vol. 26, no. 7, pp. 2344-2353, 2014

[40] B. Cho et al., "Chemical sensing of $2 \mathrm{D}$ graphene/ $\mathrm{MoS}_{2}$ heterostructure device," ACS Appl. Mater. Interfaces, vol. 7, no. 30, pp. 16775-16780, 2015.

[41] L. Staudenmaier, "Verfahren zur darstellung der graphitsäure," Berichte Deutschen Chemischen Gesellschaft, vol. 31, no. 2, pp. 1481-1487, 1898.

[42] R. Kumar et al., "Controlled density of defects assisted perforated structure in reduced graphene oxide nanosheets-palladium hybrids for enhanced ethanol electro-oxidation," Carbon, vol. 117, pp. 137-146, Jun. 2017.

[43] A. V. Alaferdov et al., "A wearable, highly stable, strain and bending sensor based on high aspect ratio graphite nanobelts," Nanotechnology, vol. 27 , no. 37, p. 375501, 2016

[44] M. Li, X. Cao, S. Zheng, and S. Qi, "Ternary composites $\mathrm{RGO} / \mathrm{MoS}_{2} @ \mathrm{Fe}_{3} \mathrm{O}_{4}$ : Synthesis and enhanced electromagnetic wave absorbing performance," J. Mater. Sci., Mater. Electron., vol. 28, no. 22, pp. 16802-16812, 2017.

[45] M. Paillet, R. Parret, J.-L. Sauvajol, and P. Colomban, "Graphene and related 2D materials: An overview of the Raman studies," J. Raman Spectrosc., vol. 49, no. 1, pp. 8-12, 2018.

[46] X. Zhang, Q.-H. Tan, J.-B. Wu, W. Shi, and P.-H. Tan, "Review on the Raman spectroscopy of different types of layered materials," Nanoscale, vol. 8, no. 12, pp. 6435-6450, 2016.

[47] M. J. Nine, M. A. Cole, L. Johnson, D. N. H. Tran, and D. Losic, "Robust superhydrophobic graphene-based composite coatings with selfcleaning and corrosion barrier properties," ACS Appl. Mater. Interfaces, vol. 7, no. 51, pp. 28482-28493, 2015.

[48] A. Dey, A. Chroneos, N. J. S. Braithwaite, R. P. Gandhiraman, and S. Krishnamurthy, "Plasma engineering of graphene," Appl. Phys. Rev., vol. 3, no. 2, p. 021301, 2016.

[49] S. K. Srivastava, B. Kartick, S. Choudhury, and M. Stamm, "Thermally fabricated $\mathrm{MoS}_{2}$-graphene hybrids as high performance anode in lithium ion battery," Mater. Chem. Phys., vol. 183, pp. 383-391, Nov. 2016.

[50] A. Khademi, R. Azimirad, A. A. Zavarian, and A. Z. Moshfegh, "Growth and field emission study of molybdenum oxide nanostars," J. Phys. Chem. C, vol. 113, no. 44, pp. 19298-19304, 2009.

[51] E. G. da Silveira Firmiano et al., "Supercapacitor electrodes obtained by directly bonding $2 \mathrm{D} \mathrm{MoS}_{2}$ on reduced graphene oxide," Adv. Energy Mater., vol. 4, no. 6, p. 1301380, 2014.

[52] Y.-T. Ho et al., "Layered $\mathrm{MoS}_{2}$ grown on $c$-sapphire by pulsed laser deposition," Phys. Status Solidi Rapid Res. Lett., vol. 9, no. 3, pp. 187-191, 2015.

[53] A. Azcatl et al., "MoS 2 functionalization for ultra-thin atomic layer deposited dielectrics," Appl. Phys. Lett., vol. 104, no. 11, p. 111601, 2014.

[54] H. Nan et al., "Strong photoluminescence enhancement of $\mathrm{MoS}_{2}$ through defect engineering and oxygen bonding," ACS Nano, vol. 8, no. 6, pp. 5738-5745, 2014.

[55] K. C. Wong, X. Lu, J. Cotter, D. T. Eadie, P. C. Wong, and K. A. R. Mitchell, "Surface and friction characterization of $\mathrm{MoS}_{2}$ and $\mathrm{WS}_{2}$ third body thin films under simulated wheel/rail rolling-sliding contact," Wear, vol. 264, nos. 7-8, pp. 526-534, 2008.

[56] J.-M. Martin, C. Grossiord, K. Varlot, B. Vacher, and J. Igarashi, "Synergistic effects in binary systems of lubricant additives: A chemical hardness approach," Tribol. Lett., vol. 8, no. 4, pp. 193-201, 2000.
[57] C. Liu et al., "Vertical single or few-layer $\mathrm{MoS}_{2}$ nanosheets rooting into $\mathrm{TiO}_{2}$ nanofibers for highly efficient photocatalytic hydrogen evolution," Appl. Catal. B, Environ., vol. 164, pp. 1-9, Mar. 2015.

[58] R. V. Gelamo, F. P. Rouxinol, C. Verissimo, A. R. Vaz M. A. B. de Moraes, and S. A. Moshkalev, "Low-temperature gas and pressure sensor based on multi-wall carbon nanotubes decorated with Ti nanoparticles," Chem. Phys. Lett., vol. 482, nos. 4-6, pp. 302-306, 2009.

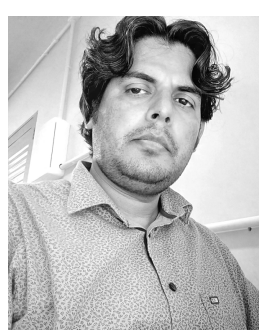

Rajesh Kumar received the Ph.D. degree in physics from the Department of Physics, Banaras Hindu University, Varanasi, India.

$\mathrm{He}$ is currently a Post-Doctoral Fellow with the Department of Physics, State University of Sao Paulo, Presidente Prudente, Brazil.

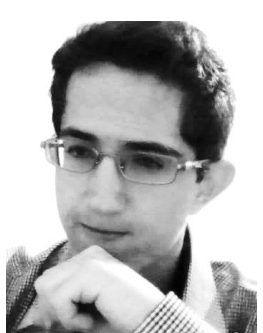

Wagner Dias, Jr. received the master's degree in materials science and technology from the State University of Sao Paulo, Presidente Prudente, Brazil, where he is currently pursuing the Ph.D. degree with the Department of Physics.

His current research interests include nanostructured ceramic materials, perovskites, microwave-assisted hydrothermal synthesis, and functional materials.

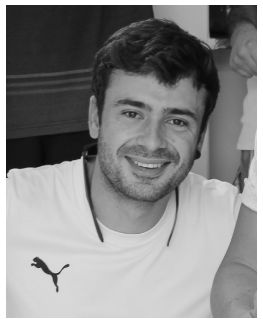

Rafael J. G. Rubira received the master's degree in materials science and technology from the State University of Sao Paulo, Bauru, Brazil. He is currently pursuing the Ph.D. degree with the Department of Physics, State University of Sao Paulo, Presidente Prudente, Brazil.

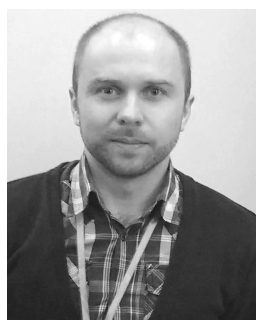

Andrei V. Alaferdov received the M.S. degree in nanotechnology in electronics and the Ph.D. degree in physics from the Lobachevsky State University of Nizhni Novgorod, Nizhny Novgorod, Russia, in 2009 and 2016, respectively.

$\mathrm{He}$ is currently a Post-Doctoral Fellow with the Center for Semiconductor Components and Nanotechnologies, State University of Campinas, Campinas, Brazil. His current research interests include the synthesis and characterization of carbon nanotubes, graphene, and hybrid structures.

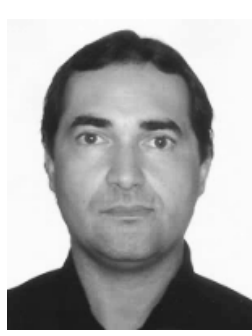

Alfredo R. Vaz received the Ph.D. degree in physics from the Institute of Physics, University of São Paulo, Brazil, in 2004.

Since 2006, he has been with the Center for Semiconductors Components and Nanotechnology, University of Campinas, Campinas, Brazil. 


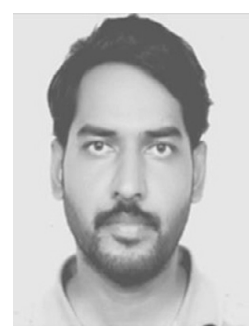

Rajesh K. Singh received the Ph.D. degree in physics from the Department of Physics, Banaras Hindu University, Varanasi, India.

$\mathrm{He}$ is currently an Assistant Professor with the School of Physical and Material Sciences, Central University of Himachal Pradesh, Dharamsala, India. His current research interests include hydrogen storage, solid oxide fuel cells, and the synthesis of carbon nanostructuresmetal/metal-oxide self-assembly and their applications in different areas.

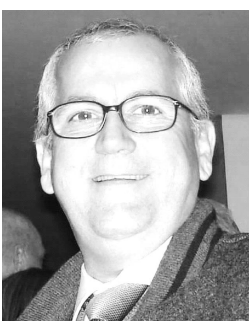

Silvio R. Teixeira received the bachelor's degree in physics, from the University of Brasília, Brasília, Brazil, and the master's degree in nuclear technology and the Ph.D. degree in applied physics from the University of Sao Paulo, Brazil.

$\mathrm{He}$ is currently a Professor of physics with the Department of Physics, State University of Sao Paulo, Presidente Prudente, Brazil. His current research interests include clay minerals, solid residues, and functional materials.

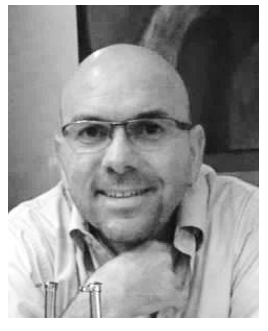

Carlos J. L. Constantino received the Ph.D. degree in physics from the Institute of Physics, University of São Paulo, Brazil.

$\mathrm{He}$ is currently a Professor of physics with the Department of Physics, State University of Sao Paulo, Presidente Prudente, Brazil. His current research interests include materials science and solid-state physics with emphasis on molecular spectroscopy, fabrication of nanostructured organic films applied to sensors, and mimetic systems of biomembranes.

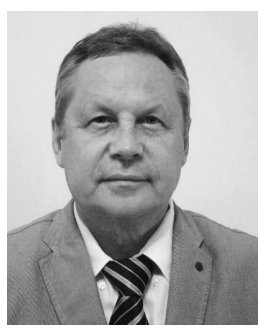

Stanislav A. Moshkalev (M'99) received the Ph.D. degree in physics from the loffe PhysicalTechnical Institute, St. Petersburg, Russia, in 1984.

$\mathrm{He}$ is currently the Director of the Center for Semiconductor Components and Nanotechnology, University of Campinas, Campinas, Brazil. He has authored over 120 articles.

Dr. Moshkalev is a member of Brazilian Physical Society and Brazilian Material Research Society. 